\title{
TEMPERATURE AND HEAT TRANSFER HISTORY OF A SOLID BODY IN A FORCED CONVECTION FLOW*
}

\author{
BY \\ N. KONOPLIV** AND E. M. SPARROW \\ University of Minnesota, Minneapolis, Minnesota
}

\begin{abstract}
Consideration is given to problems of unsteady forced convection heat transfer in the presence of either time-invariant or time-dependent surface temperatures. The transient is initiated when a solid body is exposed to a fluid having a temperature different from its own. In the first part of the paper, a solution method is developed for determining the surface heat transfer for the case of steady, uniform surface temperature. Then, attention is turned to the determination of the temperature history of non-internally-heated bodies of high thermal conductance, which lose heat by convection to the fluid environment. A numerical scheme for deducing results for the temperature history is described, while analytical expressions appropriate to the initial and quasi-steady stages of the transient are presented. Detailed consideration is given to the case of a sphere in a low Péclet number flow, for which an exact solution for the temperature history is worked out. The results from the numerical scheme are found to be in excellent agreement with those from the exact solution, while the expressions for the initial and quasi-steady stages, when taken together, serve to establish the general behavior of the solution over the entire transient period.
\end{abstract}

Introduction. In this paper, consideration is given to two classes of problems which involve time-dependent heat transfer between a solid surface and a moving fluid. One of these classes is characterized by steady, uniform surface temperatures. The other includes non-internally-heated, high-conductance solid bodies having a spatially uniform temperature which changes with time as a result of heat exchange with the fluid environment. For both classes of problems, the transient is initiated when the body is exposed to a fluid whose temperature is different from that of the body.

Upon considering the problem areas just described, it is apparent that the analysis of the first is less demanding than the analysis of the second. This is because, for the solution of problems in the first group, it is necessary to consider only the energy equation for the fluid environment (assuming that the velocity field is known). On the other hand, for problems in the second group, the energy equation for the fluid must be solved simultaneously with the energy balance for the solid.

The foregoing observation motivates one of the objectives of this research. A solution method is to be developed for determining the timewise temperature variation of a noninternally-heated solid of high conductance situated in a conducting-convecting fluid

* Received February 23, 1970.

** Present address: Polytechnic Institute of Brooklyn, Brooklyn, New York. 
environment, given the surface heat transfer solution for the same solid but with a steady, uniform surface temperature. In principle, the method is able to accommodate arbitrary body shapes.

There are a number of approaches that may be employed to generate the input information needed to apply the solution method; that is, to obtain the surface heat transfer corresponding to the condition of steady, uniform surface temperature. These approaches will be discussed in a later section. In the first section, however, a new series solution, possessing considerable generality, is presented for problems of steady, uniform surface temperature.

The second section is devoted to a description of the method for determining the timewise temperature variation of the non-internally-heated, highly conducting solid. The method makes use of integral transforms. This section also presents an account of a highly serviceable numerical inversion technique which facilitates the practical realization of the solution. In the last section of the paper, a specific problem is solved to illustrate and test the method.

Series solution for steady, uniform surface temperature. Consider a body having steady, uniform surface temperature $T_{0}$. At time $t=0$, the body is exposed to a laminar forced convection flow whose initial temperature is uniform and everywhere equal to $T_{\infty}$. The fluid freestream temperature for all subsequent times is also $T_{\infty}$. The velocity field is presumed known. For these conditions, regardless of whether the fluid velocity is steady or unsteady, the temperature field in the fluid about the sphere will undergo a timewise development from its initial uniform state. In the event that the velocity field is steady, then, after a sufficiently long time has elapsed, a thermal steady state is attained in the fluid. It is the objective of the analysis to determine the timewise variations of the local and area-integrated instantaneous heat transfer rates at the surface.

To facilitate the analysis, let $\xi, \eta, \zeta$ denote a set of dimensionless orthogonal coordinates (reference length $L$ ), such that $\eta$ is directed along the local normal to the surface, while $\xi$ and $\zeta$ lie in the surface $\eta=0$. The local instantaneous fluid temperature is represented by $T_{f^{*}}(\xi, \eta, \zeta, \tau)$, where $\tau$ is a dimensionless time. The asterisk is employed to distinguish the case of steady, uniform surface temperature that is now under study. It is also convenient to define a dimensionless fluid temperature $\theta_{f^{*}}$ as

$$
\theta_{f^{*}}(\xi, \eta, \zeta, \tau)=\left(T_{f_{*}}-T_{\infty}\right) /\left(T_{0}-T_{\infty}\right) .
$$

The first step in the analysis is to write the appropriate energy equation. For a steady, laminar boundary layer flow about a two-dimensional or axisymmetric body and for steady flow (without boundary layer assumptions) about a sphere or cylinder, the dimensionless energy equation can be written in the form

$$
\begin{aligned}
& \frac{\partial \theta_{f^{*}}}{\partial \tau}+f(\xi, \eta, \zeta) \frac{\partial \theta_{r_{*}}}{\partial \eta}=\frac{\partial^{2} \theta_{f_{*}}}{\partial \eta^{2}}+g_{1}(\eta) \frac{\partial^{2} \theta_{f_{*}}}{\partial \xi^{2}}+g_{2}(\xi, \eta) \frac{\partial^{2} \theta_{f^{*}}}{\partial \zeta^{2}} \\
& +g_{3}(\xi, \eta, \zeta) \frac{\partial \theta_{r^{*}}}{\partial \xi}+g_{4}(\xi, \eta, \zeta) \frac{\partial \theta_{r \bullet}}{\partial \zeta}
\end{aligned}
$$

with initial and boundary conditions

$$
\theta_{\rho^{*}}(\xi, \eta \neq 0, \zeta, 0)=0, \quad \theta_{\rho^{*}}(\xi, 0, \zeta, \tau)=1, \quad \theta_{\rho^{*}}(\xi, \infty, \zeta, \tau)=0 .
$$

In Eq. (2), the function $f$ includes the $\eta$-component of the fluid velocity and, depending 
on the particular case, some power of the $\eta$ coordinate which stems from the heat conduction term. The functions $g_{i}$ contain velocities and/or coordinates. Evidently, various of the $g_{i}$ are zero for two-dimensional flow, axisymmetric flow, or boundary layer flow. The $f$ and $g_{j}$ are regarded here as known functions of position.

To initiate the solution, the Laplace transform of the energy equation (2) is taken with respect to $\tau$, with $\overline{\theta_{f^{*}}}(\xi, \eta, \zeta, s)$ denoting the transform of the fluid temperature distribution. Next, to eliminate the term $\partial \overline{\theta_{f *}} / \partial \eta$ from the thus transformed energy equation, one introduces the $\bar{\psi}$ function as follows:

$$
\overline{\theta_{r}}(\xi, \eta, \zeta, s)=\frac{\bar{\psi}(\xi, \eta, \zeta, s)}{s} \exp \left[\frac{1}{2} \int_{0}^{\eta} f(\xi, \eta, \zeta) d \eta\right],
$$

and with this, the Laplace transformed version of (2) becomes

$$
\begin{aligned}
& \frac{\partial^{2} \bar{\psi}}{\partial \eta^{2}}-[s+h(\xi, \eta, \zeta)] \bar{\psi} \\
& +H\left[g_{1}(\eta) \frac{\partial^{2} \bar{\psi}}{\partial \xi^{2}}, g_{2}(\xi, \eta) \frac{\partial^{2} \bar{\psi}}{\partial \zeta^{2}}, h_{1}(\xi, \eta, \zeta) \frac{\partial \bar{\psi}}{\partial \xi}, h_{2}(\xi, \eta, \zeta) \frac{\partial \bar{\psi}}{\partial \zeta}\right]=0 .
\end{aligned}
$$

In addition, under the transformation, the boundary conditions become

$$
\bar{\psi}(\xi, 0, \zeta, s)=1, \quad \bar{\psi}(\xi, \infty, \zeta, s)=0 .
$$

The functions $h, h_{1}$, and $h_{2}$ are related to the $f$ and $g_{j}$ of Eq. (2) and can, therefore, be regarded as known, as can the functional form $H$.

A series solution of (5) and (6) will now be sought which is valid for large values of $s$ (large $s$ corresponds to small $\tau$ ). This problem resembles that of the Liouville type [1] and, correspondingly, an appropriate form for the series solution is assumed to be

$$
\bar{\psi}(\xi, \eta, \zeta, s)=\exp \left(-s^{1 / 2} \eta\right) \sum_{i=0}^{\infty} y_{i}(\xi, \eta, \zeta) s^{-i / 2}
$$

from which it follows, in conjunction with (6), that

$$
y_{0}(\xi, 0, \zeta)=1, \quad y_{i}(\xi, 0, \zeta)=0 \text { for } i \geq 1 .
$$

The substitution of (7) into (5) and subsequent grouping of terms according to powers of $s$ yjelds

$$
y_{0}(\xi, \eta, \zeta)=1
$$

where the boundary condition (8) has been satisfied. For the other $y_{i}, i \geq 1$, one finds

$$
\begin{aligned}
\frac{\partial y_{i}}{\partial \eta}= & \frac{1}{2} \frac{\partial^{2} y_{i-1}}{\partial \eta^{2}}-\frac{1}{2} h(\xi, \eta, \zeta) y_{i-1} \\
& +\frac{1}{2} H\left[g_{1}(\eta) \frac{\partial^{2} y_{i-1}}{\partial \xi^{2}}, g_{2}(\xi, \eta) \frac{\partial^{2} y_{i-1}}{\partial \zeta^{2}}, h_{1}(\xi, \eta, \zeta) \frac{\partial y_{i-1}}{\partial \xi}, h_{2}(\xi, \eta, \zeta) \frac{\partial y_{i-1}}{\partial \zeta}\right]=0 .
\end{aligned}
$$

Of particular interest to the present study are the derivatives $\left(\partial y_{i} / \partial \eta\right)_{\eta=0}$, which are involved in the determination of the local surface heat flux $q$. These derivatives can be determined from Eq. (10). Then, by employing Fourier's law, $q=-\left(k_{f} / L\right)(\partial T / \partial \eta)_{\eta=0}$, in conjunction with Eqs. (1), (4), (6), (7), (8), (9), and (10), one obtains

$$
\frac{\overline{q_{*}} L}{k_{f}\left(T_{0}-T_{\infty}\right)}=\frac{1}{s^{1 / 2}}-\frac{f(\xi, 0, \zeta)}{2 s}+\frac{h(\xi, 0, \zeta)}{2 s^{3 / 2}}+\frac{1}{4 s^{2}}\left(\frac{\partial h}{\partial \eta}\right)_{\xi, 0,5}-\sum_{i=3}^{\infty} \frac{1}{s^{1+i / 2}}\left(\frac{\partial y_{i}}{\partial \eta}\right)_{\xi, 0, \zeta}
$$


or, after inverse transformation into the time domain,

$$
\frac{q_{*} L}{k_{f}\left(T_{0}-T_{\infty}\right)}=\frac{1}{(\pi \tau)^{1 / 2}}-\frac{f(\xi, 0, \zeta)}{2}+h(\xi, 0, \zeta)\left(\frac{\tau}{\pi}\right)^{1 / 2}+\frac{1}{4}\left(\frac{\partial h}{\partial \eta}\right)_{\xi, 0, \zeta} \tau-\cdots .
$$

It can be verified that $f(\xi, 0, \zeta)$ and $h(\xi, 0, \zeta)$ do not contain any convective ingredients in the absence of surface mass transfer. Therefore, the first three terms are purely conductive, and convection enters in the fourth or subsequent terms.

For later application in the next section of the paper, the Laplace transform of the surface-integrated heat transfer rate $Q=\int_{A} q d A$ is needed. After integration of Eq. (11) over the surface and introduction of the Nusselt number, there follows

$$
\begin{aligned}
\overline{N u_{*}}(s) & \left.=\overline{\left(Q_{*}\right.} / A\right)(2 L) /\left(k_{f}\left(T_{0}-T_{\infty}\right)\right) \\
& =\frac{2}{s^{1 / 2}}+\frac{1}{A} \int_{A}\left[-\frac{f}{s}+\frac{h}{s^{3 / 2}}+\frac{1}{2 s^{2}} \frac{\partial h}{\partial \eta}\right]_{\xi, 0, \zeta} d A+O\left(s^{-5 / 2}\right)
\end{aligned}
$$

with a corresponding expression in the time domain.

As an illustration of the foregoing, consider flow over a sphere without boundary layer approximation. For this case, the local heat flux representation (12) reduces to (with $L=r_{0}$, the sphere radius)

$$
\frac{q_{*} r_{0}}{k_{f}\left(T_{0}-T_{\infty}\right)}=\frac{1}{(\pi \tau)^{1 / 2}}+1-\frac{P e}{16}\left(\frac{\partial^{2} u_{\eta}}{\partial \eta^{2}}\right)_{\varepsilon, 0,5} \tau+\cdots
$$

In (14), $P e$ is the Péclet number $\left(=2 r_{0} U_{\infty} / \alpha_{f}\right), \tau$ is the Fourier number $\left(=\alpha_{f} t / r_{0}^{2}\right), u_{\eta}$ is the dimensionless radial velocity (relative to freestream $\left.U_{\infty}\right), \eta=\left(r-r_{0}\right) / r_{0}$, and $\xi$ and $\zeta$ are the cone and azimuth angles respectively. It is seen that the first influence of convection is manifested via the wall value of the derivative $\partial^{2} u_{\eta} / \partial \eta^{2}$, the magnitude of which generally depends on surface location. This derivative can be evaluated for the various flow regimes for which velocity solutions exist, e.g. Stokes or Oseen.

The foregoing solution method was limited to laminar external flows. Provided that the eddy conductivity for heat is a known function of position, then it appears possible, in principle, to extend the method to turbulent external flows.

Now that the development of the series solution for the case of steady, uniform surface temperature is complete, attention can be turned to the main concern of the paper, that is, solids with time-varying temperatures.

Temperature history of a highly conducting solid. In this section, a solution method is presented for determining the timewise temperature variation of a solid situated in a forced convection flow. The method makes use of the heat transfer results corresponding to the condition that the solid is maintained at a steady, uniform surface temperature.

The temperature history of the solid is controlled by an instantaneous balance between the conductive-convective heat transfer to (or from) the fluid environment and the change of internal energy of the solid. It is postulated that the conductance of the solid is sufficiently high so that its temperature is spatially uniform at any instant of time. There are no internal sources of heat within the solid. Initially, at time $t=0$, the solid and fluid are at different uniform temperatures, $T_{0}$ and $T_{\infty}$, respectively. The fluid freestream temperature is $T_{\infty}$ for all time.

To begin the analysis, dimensionless temperatures are defined as

$$
\theta_{s}(\tau)=\left(T_{s}-T_{\infty}\right) /\left(T_{0}-T_{\infty}\right), \quad \theta_{f}(\xi, \eta, \zeta, \tau)=\left(T_{f}-T_{\infty}\right) /\left(T_{0}-T_{\infty}\right),
$$


where $T_{s}(\tau)$ is the spatially uniform temperature of the solid and $T_{f}(\xi, \eta, \zeta, \tau)$ is the local temperature of the fluid. Temperature continuity at the surface of the solid requires that

$$
\theta_{s}(\tau)=\theta_{f}(\xi, 0, \zeta, \tau)
$$

for all $\tau>0$.

At any instant of time, the rate of change of the internal energy of the solid is equal to the rate of conductive-convective heat transfer at the surface, so that

$$
-\rho_{s} c_{s} V \frac{d T_{s}}{d t}=Q=-\int_{A} \frac{k_{f}}{L}\left(\frac{\partial T_{f}}{\partial \eta}\right)_{\eta-0} d A,
$$

in which $V$ is the volume of the solid. When Eq. (17) is recast into dimensionless form, one gets

$$
\nu \frac{d \theta_{s}}{d \tau}=\frac{2}{A} \int_{A}\left(\frac{\partial \theta_{f}}{\partial \eta}\right)_{\eta=0} d A, \quad \nu=\frac{2 V}{L A} \frac{\rho_{s} c_{s}}{\rho_{f} c_{f}} .
$$

Attention is now turned to the surface derivative of the fluid temperature, $\partial \theta_{f} / \partial \eta$, and its elimination from the problem. To this end, we note that the temperature $\theta_{f}(\xi, \eta$, $\zeta, \tau)$ at any point in the fluid can be represented, via Duhamel's integral, in terms of the temperature solution $\theta_{f *}(\xi, \eta, \zeta, \tau)$ for the case of steady, uniform surface temperature, that is

$$
\theta_{f}(\xi, \eta, \zeta, \tau)=\int_{0}^{\tau} \theta_{s}(\lambda) \frac{\partial}{\partial \tau}\left[\theta_{f^{*}}(\xi, \eta, \zeta, \tau-\lambda)\right] d \lambda .
$$

The application of Duhamel's integral is well established in heat conduction problems, but its use in convection problems is much less common. Integration by parts of the right-hand side of Eq. (19) and application of the condition $\theta_{s}(0)=1$ lead to

$$
\theta_{f}(\xi, \eta, \zeta, \tau)=\theta_{f^{*}}(\xi, \eta, \zeta, \tau)+\int_{0}^{\tau} \frac{d \theta_{s}(\lambda)}{d \lambda} \theta_{f^{*}}(\xi, \eta, \zeta, \tau-\lambda) d \lambda,
$$

which satisfies the initial and boundary conditions on $\theta_{f}$. The elimination of $\theta_{f}$ and $\partial \theta_{f} / \partial \eta$ between Eqs. (18) and (20) yields

$$
\nu \frac{d \theta_{s}}{d \tau}=-N u_{*}(\tau)-\int_{0}^{\tau} \frac{d \theta_{s}(\lambda)}{d \lambda} N u_{*}(\tau-\lambda) d \lambda,
$$

where

$$
N u_{*}(\tau)=\frac{\left(Q_{*} / A\right)(2 L)}{k_{f}\left(T_{0}-T_{\infty}\right)}=-\frac{2}{A} \int_{A}\left(\frac{\partial \theta_{f *}}{\partial \eta}\right)_{\eta=0} d A .
$$

The quantity $N u_{*}(\tau)$ represents the Nusselt number for the case of steady, uniform surface temperature.

Eq. (21) is an integral equation for the time-derivative of the temperature of the solid. The solution of (21) might be attempted by the application of various available methods. Here, a different approach, based on integral transforms, is employed. As will be demonstrated in the next subsection, one of the advantages of the present approach is that input information on $\overline{N u_{*}}(s)$ (Laplace transform of $N u_{*}(\tau)$ ) is needed only for a discrete number of values of $s$. 
The basic equation for the new solution method is deduced by taking the Laplace, transform of Eq. (21), which vields

$$
\bar{\theta}_{s}(s)=\frac{v}{\nu s+\overline{s i u_{*}}(s)},
$$

where $\bar{\theta}_{s}(s)$ and $\overline{N u_{*}}(s)$ are the transformed counterparts of $\theta_{s}(\tau)$ and $N u_{*}(\tau)$. Upon inspection of Eq. (23), it is seen that the nature of the task of determining $\theta_{s}(\tau)$ is altogether different from that embodied in Eq. (21). According to Eq. (23), the input information is fed into the problem in terms of $\overline{N u_{*}}(s)$, and the major task is to perform the inverse Laplace transform of the right-hand side. The inversion can expeditiously be carried out numerically as described later, but in some cases is capable of being performed analytically. On the other hand, the task embodied in Eq. (21) is the solution of an integral equation, followed by integration to obtain $\theta_{s}(\tau)$.

Relevant to Eq. (23), it is appropriate to discuss (a) methods for determining the input function $\overline{N u_{*}}(s)$ and (b) methods for inverting the equation in order to find $\theta_{s}(\tau)$.

With respect to the function $\overline{N u_{*}}(s)$, there are several approaches which appear to be serviceable. One is to perform a finite-difference solution of the Laplace-transformed energy equation. Such a transformed energy equation resembles that for steady-state convection with a heat source proportional to $s \overline{\theta_{*}}$. The numerical solution need be performed for only a small number of discrete values of $s$. Another way of generating $\overline{N u_{*}}(s)$ is by a series solution such as that given in an earlier section of this paper. For solids with relatively small heat capacity (rapid transients for $\theta_{s}(\tau)$ ), the $\theta_{s}(\tau)$ results which are deduced from a series input for $\overline{N u_{*}}(s)$ will cover most of the transient period. On the other hand, for solids with relatively large heat capacity, the series input provides a small time solution for $\theta_{s}(\tau)$.

A third approach to obtaining $\overline{N u_{*}}(s)$ is to take

$$
\overline{N u_{*}}(s)=N u_{*}(\infty) / s
$$

where $N u_{*}(\infty)$ is the steady-state Nusselt number corresponding to the case of steady, uniform surface temperature. This approach is relevant to the situation in which the convective heat transfer is essentially quasi-steady. As is shown later, the $\theta_{s}(\tau)$ deduced by using (24) as input is of satisfactory accuracy over most of the transient period for solids of relatively high heat capacity and otherwise serves as a large time solution. In any case, Eq. (24), taken together with the aforementioned series input for $\overline{N u_{*}}(s)$, provides an effective means of establishing the behavior of $\theta_{s}(\tau)$ over the entire transient period. Finally, in some cases, $\overline{N u_{*}}(s)$ may be obtained by an exact solution (see, for example, [2]).

Attention is now turned to the inverse transformation of Eq. (23) to determine $\theta_{s}(\tau)$. A highly serviceable numerical inversion technique can be utilized for this purpose. This technique, which is described in the next subsection, appears to be quite promising, but it has yet to be widely exploited in heat transfer applications. The inverse transform can be carried out exactly to yield the aforementioned initial-stage and quasi-steady solutions for $\theta_{s}(\tau)$, a task accomplished in the second subsection. Also, in certain special cases, the inverse transformation can be performed exactly for all time, as is exemplified in the last section of the paper.

Determination of $\theta_{s}(\tau)$ by numerical inversion. If $\overline{N u_{*}}$ is a known function of $s$ then from Eq. (23), $\bar{\theta}_{s}(s)$ is also a known function. The relationship between the known 
$\bar{\theta}_{s}(s)$ and the unknown time-dependent temperature $\theta_{s}(\tau)$ is embodied in the definition of the Laplace transform

$$
\bar{\theta}_{s}(s)=\int_{0}^{\infty} \exp (-s \tau) \theta_{s}(\tau) d \tau
$$

Introduction into (25) of a change in the time variable from $\tau$ to $\Lambda$

$$
\tau=-a \ln \Lambda / b
$$

along with a new unknown function $\hat{\theta}_{s}$ which is related to $\theta_{s}$ by

$$
\theta_{s}(\tau)=b \exp (c \tau) \hat{\theta}_{s}(b \tau)
$$

leads to the following:

$$
\bar{\theta}_{s}(s b+c)=a \int_{0}^{1} \Lambda^{a s-1} \hat{\theta}_{s}(-a \ln \Lambda) d \Lambda .
$$

In (28), $a, b$, and $c$ are prescribable real constants selected to facilitate stability of the solution. Although there exists no theoretical prescription for an optimum choice of $a, b$, and $c[3]$, some pertinent observations about their selection, drawn from the authors' experience, will be given later.

Eq. (28) is an integral equation for $\hat{\theta}_{s}$. Its solution is found here with the help of the Gaussian quadrature integration formula. For a preselected number of points $N$ in the interval $0 \leq \Lambda \leq 1$, the integral is approximated by $N$ terms, each term containing a known weight $w_{i}$ and known abscissa $\Lambda_{i}$, where $i=1,2, \cdots, N$. Experience suggests that a maximum value of 10 for $N$ is quite sufficient for most applications. The integration procedure introduces $N$ unknowns, $\hat{\theta}_{s}\left(-a \ln \Lambda_{i}\right), i=1,2, \cdots, N$. Since one is at liberty to assign $N$ real values to $s$, say $s_{i}, j=1,2, \cdots, N$, the $N$ unknown values of $\hat{\theta}_{s}$ are thus found with the aid of $N$ linear equations,

$$
\bar{\theta}_{s}\left(s_{i} b+c\right)=a \sum_{i=1}^{N} w_{i} \Lambda_{i}^{a s,-1} \hat{\theta}_{s}\left(-a \ln \Lambda_{i}\right), \quad j=1,2, \cdots, N .
$$

Once the $\hat{\theta}_{s}$ are determined, the dimensionless temperature $\theta_{s}(\tau)$ of the solid is found with the help of Eqs. (26) and (27).

The authors have inverted numerous functions from the complex s-plane. In all of the cases investigated, the $s_{i}$ which appear in Eq. (29) were selected to be $s_{i}=0,1, \cdots, 9$. The constant $a$, in Eqs. (26) and (29), was taken as one. A numerical value of $b$ was chosen in order to provide the desired range for $\tau$, as calculated from $\tau=-a \ln \Lambda / b$ and from the $\Lambda_{i}$ of the Gaussian integration procedure. The remaining constant $c$ was selected by trial and error, in such a way that the $\theta_{s}(\tau)$ results were in agreement for more than one value of $b$.

Solutions for the initial and quasi-steady stages. The series solution for $\overline{N u_{*}}(s)$, Eq. (13), can be used in conjunction with Eq. (23) to generate a representation for $\theta_{s}(\tau)$ for the initial stage of the transient period. If the $1 / s^{1 / 2}$ and $1 / s$ terms of the series are employed, one finds, after inverse transformation, respectively for $f(\xi, 0, \zeta) \neq 0$ and $=0$,

$$
\begin{aligned}
\theta_{s}(\tau) & =\frac{4 \nu}{\pi} \int_{0}^{\infty} \exp \left(-\chi^{2} \tau\right)\left[\left(f(\xi, 0, \zeta)+\nu \chi^{2}\right)^{2}+4 \chi^{2}\right]^{-1} \chi^{2} d \chi \\
& =\exp (4 \tau / \nu) \operatorname{erfc}(2 \sqrt{\tau} / \nu) .
\end{aligned}
$$


Insights into the range of $\tau$ for which Eq. (30) is applicable, with particular reference to the influence of heat capacity, will become evident during the presentation of results for the forthcoming example problem.

To obtain the quasi-steady representation, $\overline{N u_{*}}(s)$ from (24) is introduced into (23). This yields, after transformation,

$$
\theta_{s}(\tau)=\exp \left[-N u_{*}(\infty) \tau / \nu\right] .
$$

Further insights into the utility of (31) will be provided by the example problem.

Sphere in a uniform velocity field. In order to amplify and illuminate the solution method described in the preceding portion of the paper, application is made to the problem of a sphere situated in an everywhere uniform velocity field. In the range of low Péclet numbers, it was shown in [2] that the $N u_{*}(\tau)$ results from this model are in good agreement with those corresponding to the Stokes velocity field.

This problem admits an exact solution for the time history $\theta_{s}(\tau)$ of the highly conducting sphere. In addition to those of the exact solution, results for $\theta_{s}(\tau)$ are also obtained by numerical inversion and from the initial-stage and quasi-steady representations. Comparisons with the exact solution provide insights into the utility of these other approaches for determining $\theta_{s}(\tau)$.

The initial and boundary conditions for the temperature are the same as those stated earlier in the paper.

Attention is first focused on the case of steady, uniform surface temperature, for which the solution is given in [2] for the range of low Péclet numbers. The closed-form representation for $\overline{\mathrm{Nu}_{*}}(s)$, taken from [2] and reproduced here for convenience, is

$$
\overline{N u_{*}}(s)=\frac{1}{s}-\frac{4 \pi}{P e} \frac{\tilde{s}^{1 / 2}}{s} \sum_{n=0}^{\infty}(-1)^{n}(2 n+1) \bar{I}_{n+1 / 2}^{2}\left(\frac{P e}{4}\right) K_{n+1 / 2}^{\prime}\left(\tilde{s}^{1 / 2}\right) / K_{n+1 / 2}\left(\tilde{s}^{1 / 2}\right)
$$

where $\tilde{s}=s+P e^{2} / 16$. The Péclet number $P e$ is equal to $2 r_{0} U_{\infty} / \alpha_{f} . I$ and $K$ are the modified Bessel functions, respectively of the first and second kind, while $K^{\prime}$ is the derivative of $K$ with respect to its argument.

Next, consideration is given to determining the exact solution for $\theta_{s}(\tau)$. To this end, the $\overline{N u_{*}}(s)$ of Eq. (32) is introduced into (23), giving

$$
\bar{\theta}_{s}(s)=\nu /\left(\nu \tilde{s}+B+\widetilde{s}^{1 / 2} \sum_{n=0}^{\infty} B_{n} K_{n+3 / 2}\left(\tilde{s}^{1 / 2}\right) / K_{n+1 / 2}\left(\tilde{s}^{1 / 2}\right)\right)
$$

where now

$$
\nu=\left(\frac{2}{3}\right)\left(\rho_{s} c_{s} / \rho_{f} c_{f}\right)
$$

and

$$
\begin{aligned}
& B=1-\nu \frac{P e^{2}}{16}-\frac{2 \pi}{P e} \sum_{n=0}^{\infty}(-1)^{n}(2 n+1)^{2} I_{n+1 / 2}^{2}\left(\frac{P e}{4}\right) \\
& B_{n}=\frac{4 \pi}{P e}(-1)^{n}(2 n+1) I_{n+1 / 2}^{2}\left(\frac{P e}{4}\right) .
\end{aligned}
$$

The inverse transform of (33) was obtained by contour integration performed in the complex $\tilde{s}$ plane. Upon inversion, the result is multiplied by $\exp \left(-P e^{2} \tau / 16\right)$ to give $\theta_{s}(\tau)$. Since the denominator of (33) possesses a branch point at $\tilde{s}=0$, the contour for the inversion integral does not cross the negative real axis nor the origin. It is a standard 
contour, arising in other applications, and is depicted, for example, by Carslaw and Jaeger [4, p. 303]. In addition, the denominator may possess, at most, a pole which is denoted by $\widetilde{S}_{p}$. If it exists, the pole is of order one and is located on the positive real axis. Values of $\widetilde{S}_{p}$ and the corresponding residues Res of the right-hand side of (33) are presented in Fig. 1 as a function of the heat capacity ratio $\rho_{s} c_{s} / \rho_{f} c_{f}$, with the Péclet number $P e$ as curve parameter.

The end result of the inverse transformation is

$$
\begin{aligned}
\theta_{*}(\tau)=\frac{2 v}{\pi} \exp \left(-\frac{P e^{2}}{16} \tau\right) & \int_{0}^{\tau} \exp \left(-\chi^{2} \tau\right) \\
& \cdot \frac{\chi^{2} \sum_{n=0}^{\infty} B_{n} \Gamma_{n}(\chi)}{\chi^{2}\left[\sum_{n=0}^{\infty} B_{n} \Gamma_{n}(\chi)\right]^{2}+\left[\nu \chi^{2}-B-\chi \sum_{n=0}^{\infty} B_{n} \Delta_{n}(\chi)\right]^{2}} d \chi \\
+ & \operatorname{Res} \cdot \exp \left(\tilde{S}_{p} \tau-\frac{P e^{2}}{16} \tau\right),
\end{aligned}
$$

in which $\Gamma_{n}$ and $\Delta_{n}$ represent expressions which involve the Bessel functions $J$ and $Y$, that is,

$$
\Gamma_{n}(\chi)=\frac{J_{n+3 / 2}(\chi) Y_{n+1 / 2}(\chi)-J_{n+1 / 2}(\chi) Y_{n+3 / 2}(\underline{)})}{J_{n+1 / 2}^{2}(\chi)+Y_{n+1 / 2}^{2}(\chi)},
$$

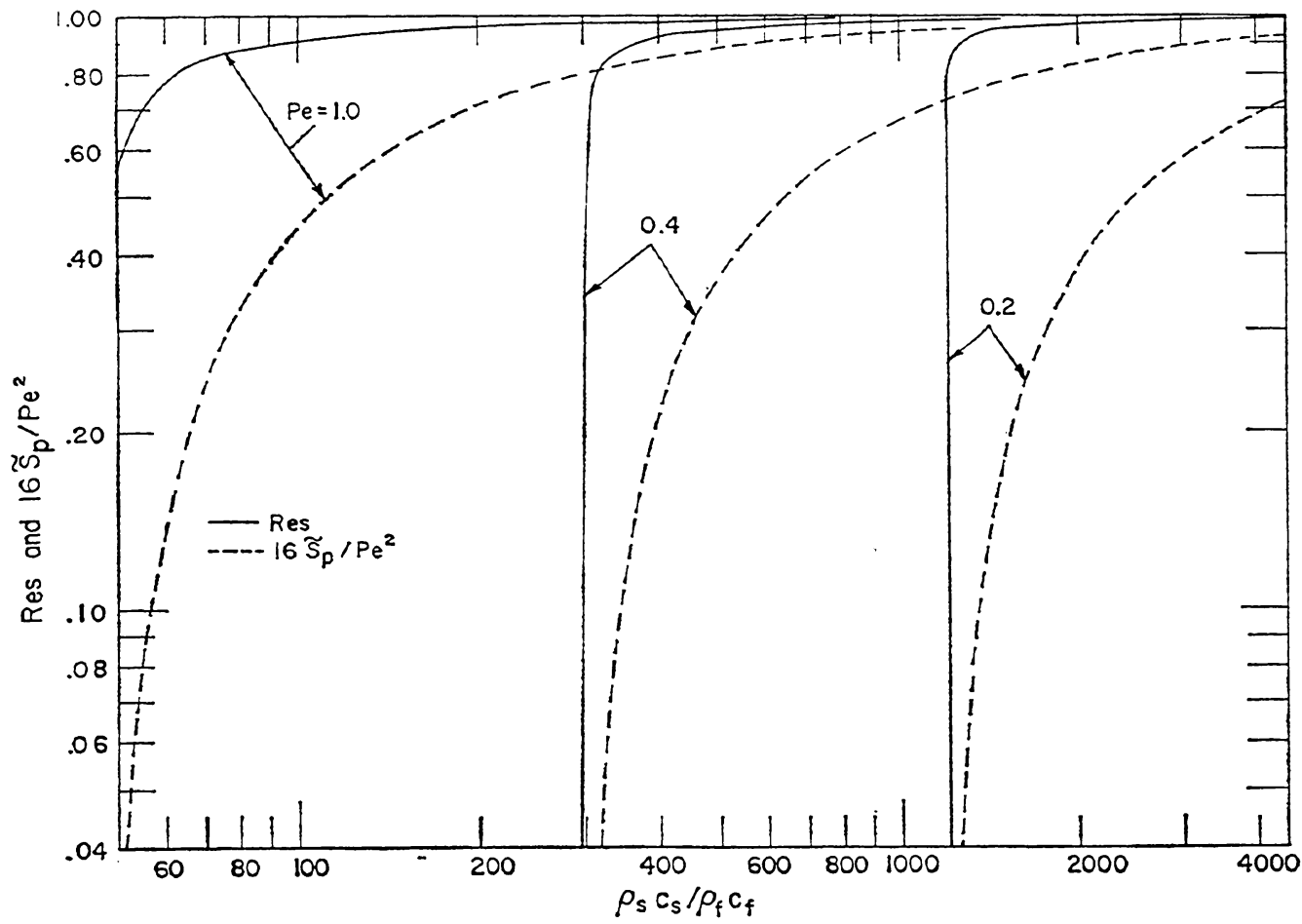

FIG. 1. The pole $\tilde{S}_{p}$ and the corresponding residues. 


$$
\Delta_{n}(\chi)=\frac{J_{n+3 / 2}(\chi)}{J_{n+1 / 2}(\chi)+Y_{n+3 / 2}(\chi) Y_{n+1 / 2}(\chi)} .
$$

Temperature histories, evaluated from the exact solution (36), are plotted as solid lines in Fig. 2 for parametric values of the ratio $\rho_{s} c_{s} / \rho_{f} c_{f}$ ranging from 10 to 4,500 and for a Péclet number equal to unity. The figure shows that the extent of the transient period increases significantly as the heat capacity of the solid grows larger relative to that of the fluid. This trend is reasonable on physical grounds.

The numerical inversion technique described in the preceding section of the paper was applied to obtain $\theta_{8}(\tau)$ results from Eq. (33). The results from the numerical inversion, shown as darkened points in Fig. 2, are in excellent agreement with those from the exact solution. This level of agreement helps to establish the numerical inversion technique as a viable approach for obtaining results of good accuracy for the temperature history.

Results for $\theta_{s}(\tau)$ have also been evaluated from the initial-stage and quasi-steady solutions, Eqs. (30) and (31) respectively. In the first of Eqs. (30), the function $f(\xi$, $0, \zeta)=-2$, while in Eq. $(31), N u_{*}(\infty)=2.46 \cdots 6$, which corresponds to the steadystate Nusselt number for a sphere in a uniform velocity field with $P e=1$. The initialstage and quasi-steady solutions are respectively depicted in the figure by dot-dashed and dashed lines.

Inspection of the figure reveals that for the smaller values of $\rho_{s} c_{s} / \rho_{f} c_{f}$, the initialstage solution provides an accurate representation of $\theta_{s}(\tau)$ over most of the transient period, and only at the larger $\rho_{s} c_{s} / \rho_{f} c_{f}$ is its accuracy limited to the first part of the transient period. In general, for a given $\rho_{s} c_{s} / \rho_{f} c_{f}$, this solution is valid for $\tau<\left[1.7\left(\rho_{s} c_{s} / \rho_{f} c_{f}\right)\right]^{1 / 2}$. On the other hand, the quasi-steady solution is virtually indistinguishable from the exact solution over the entire transient period when $\rho_{s} c_{s} / \rho_{f} c_{f}$ is large; at the smaller values of $\rho_{s} c_{s} / \rho_{f} c_{f}$, its validity is confined to the final phases of the

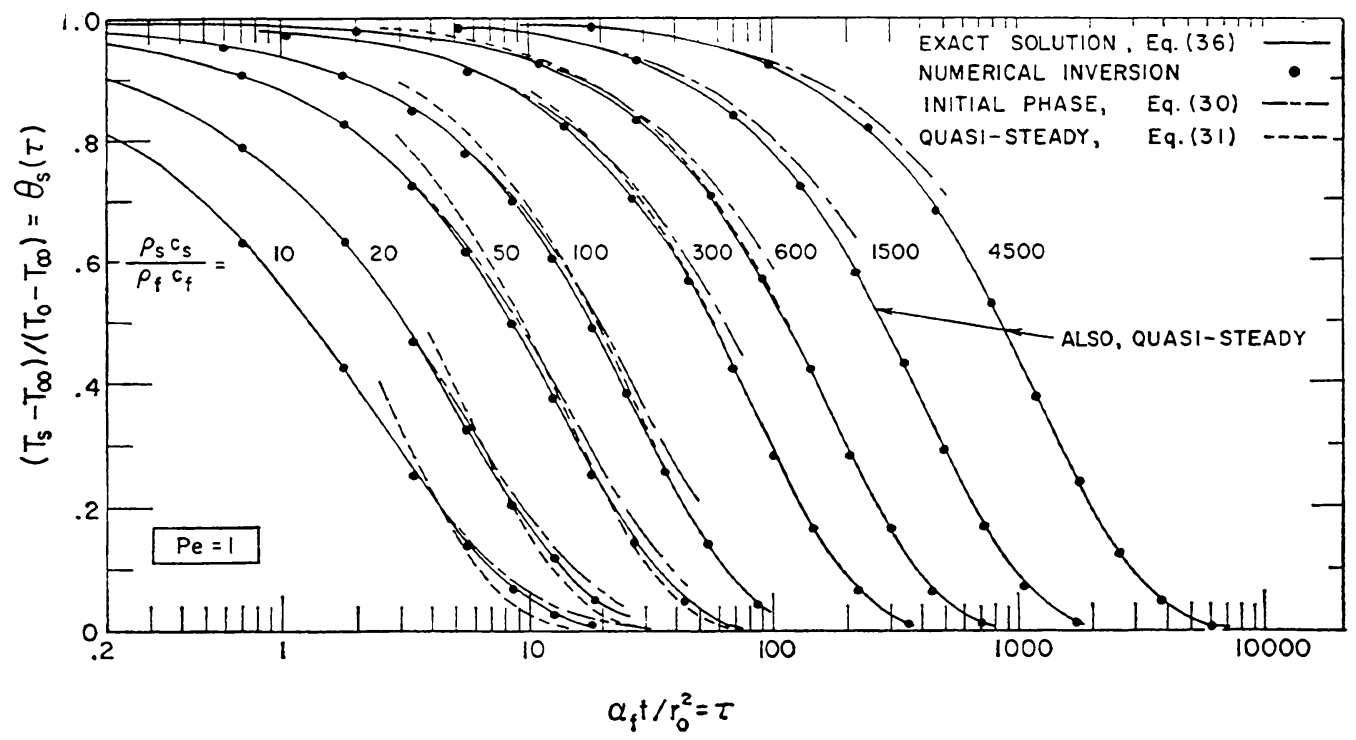

FIG. 2. Temperature histories for a sphere in a uniform velocity field. 
transient. It is evident that, taken together, the initial-stage and quasi-steady solutions effectively serve to establish the behavior of $\theta_{s}(\tau)$ over the entire transient period. Acknowledgment. Fellowship support accorded to N. Konopliv by the Whirlpool Corporation is gratefully acknowledged.

\section{REFERENCES}

[1] A. Erdelyi, Asymptotic expansions, Dover, New York, 1956

[2] N. Konopliv and E. M. Sparrow, Transient heat transfer between a moving sphere and a fluid, Fourth International Heat Transfer Conference III, FC 7.4, Paris-Versailles, 1970

[3] R. E. Bellman, K. E. Kalaba and J. Lochet, Numerical inversion of the Laplace transform, American Elsevier, New York, 1966

[4] H. S. Carslaw and J. C. Jaeger, Conduction of heat in solids, 2nd ed., Clarendon Press, Oxford, 1.959 